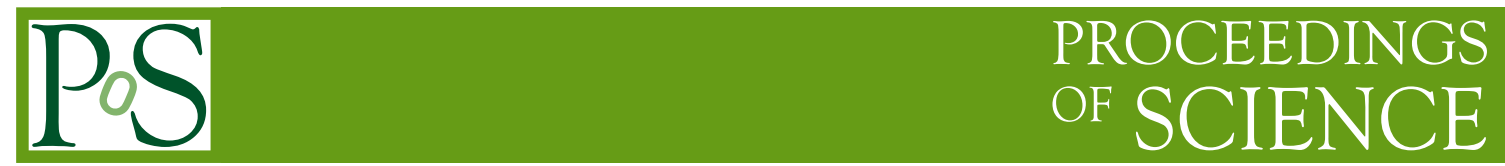

\title{
Top-pair production and decay at the LC
}

\author{
Vladimir Kuksa* \\ Institute of Physics, Southern Federal University, Russia \\ E-mail: kuksa@list.ru
}

\section{Roman Pasechnik}

Theoretical High Energy Physics, Department of Astronomy and Theoretical Physics, Lund

University, Sweden

E-mail: roman.pasechnik@thep.lu.se

\section{Dima Vlasenko}

Institute of Physics, Southern Federal University, Russia

E-mail: vlasenko@list.ru

Top quark pairs production and decay are considered in the framework of the smeared-mass unstable particles model. The results are in good agreement with the previous ones in the literature. Suggested approach significantly simplifies calculations compared to the standard perturbative one.

The XXth International Workshop on High Energy Physics and Quantum Field Theory September 24-October 1, 2011

Sochi Russia

${ }^{*}$ Speaker. 


\section{Introduction}

The top pair production and decay are the key processes for precision tests of the Standard Model (SM) (see e.g. Ref. [1] and references therein). Nowadays, the biggest attention is paid to the process of the top quark production at the LHC (see e.g. Refs. [2, 3]). However, the highest precision measurements of the top quark properties can best be reached at the future Linear Collider (LC) which operates in a clean experimental environment. The top quark physics is one of the most interesting and challenging targets in future $e^{+} e^{-}$or $\mu^{+} \mu^{-}$LC experiments [4].

The cross-sections of the processes $e^{+} e^{-} \rightarrow b \bar{b} u \bar{d} \mu^{-} \bar{v}_{\mu}$ and $e^{+} e^{-} \rightarrow b \bar{b} 4 q$ were calculated in Refs. [5, 6] and [7], respectively, in the Born approximation. Other exclusive reactions with $b \bar{b} d \bar{u} \mu^{+} v_{\mu}, b \bar{b} c \bar{s} d \bar{u}$ and $b \bar{b} \mu^{+} v_{\mu} \tau^{-} \bar{v}_{\tau}$ final states were considered in Ref. [4]. In particular, it was shown that the contribution of the top-pair signal $e^{+} e^{-} \rightarrow t^{*} \bar{t}^{*} \rightarrow b \bar{b} 4 f$ is dominant, but the background (caused by one-resonant or non-resonant diagrams) is quite significant. However, it can be drastically decreased by applying cuts on the appropriate invariant masses.

One-loop EW and QCD corrections for the reaction $e^{+} e^{-} \rightarrow t \bar{t}$ were previously calculated in many papers (see e.g. Ref. [8] and references therein). Concerning radiative corrections (RC) to reaction $e^{+} e^{-} \rightarrow b \bar{b} 4 f$ with six-fermion final states, the situation is more complicated and less advanced [8]. The most detailed analysis of the exclusive reactions $e^{+} e^{-} \rightarrow b \bar{b} \mu^{+} v_{\mu} \mu^{-} \bar{v}^{\mu}$ and $e^{+} e^{-} \rightarrow b \bar{b} d \bar{u} \mu^{-} \bar{v}^{\mu}$ was performed in Ref. [8]. There, the cross-sections were calculated with an account of the leading radiative corrections, such as the initial state radiation (ISR) and factorizable EW corrections to the on-shell top-pair production, decay of the top into $b W$ and to the subsequent decays of the $W$-bosons.

In this work, we consider reactions like $e^{+} e^{-} \rightarrow t^{*} \bar{t}^{*} \rightarrow b \bar{b} 4 f$ with any four-fermion final states $4 f$. The analysis is performed in the framework of the smeared mass unstable particles model (SMUP model) $[9,10]$. Due to exact factorization at intermediate $t, \bar{t}$ and $W^{+}, W^{-}$, the cross-section can be represented in a simple analytical form which is convenient for analysis. In our calculations, we take into account NLO radiative EW and QCD factorizable corrections. The results are compared with ones calculated by using the standard methods [8]. It was shown that in the Born approximation the results coincide with a rather high precision, and deviations of the higher-order corrected results from the standard ones are at the percentage level or less. So, the suggested approach can be applied for a fast preliminary analysis of various complicated processes in the Standard Model and beyond.

\section{Model cross-section of the top-pair production and decay at the tree level}

The process of top-pair production with subsequent decay $e^{+} e^{-} \rightarrow t^{*} t^{*} \rightarrow b \bar{b} W^{+} W^{-} \rightarrow b \bar{b} 4 f$ is schematically represented in Fig. 1. The full process contains two steps with unstable intermediate time-like states, namely, $t, \bar{t}$ and $W^{+}, W^{-}$states. In this case, as was shown in Ref. [10], the double factorization effects take place. Due to these effects the full process can be divided into three stages: $e^{+} e^{-} \rightarrow t^{*} \bar{t}^{*}, t^{*} \bar{t}^{*} \rightarrow b \bar{b} W^{+} W^{-}$and $W^{+} W^{-} \rightarrow 4 f$.

The SMUP model cross-section of the first reaction $e^{+} e^{-} \rightarrow t^{*} t^{*}$ can be written as [9]

$$
\sigma\left(e^{+} e^{-} \rightarrow t^{*} \bar{t}^{*}\right)=\int_{m_{0}^{2}}^{s} \int_{m_{0}^{2}}^{\left(\sqrt{s}-m_{1}\right)^{2}} \sigma\left(e^{+} e^{-} \rightarrow t\left(m_{1}\right) \bar{t}\left(m_{2}\right)\right) \rho_{t}\left(m_{1}\right) \rho_{t}\left(m_{2}\right) d m_{1}^{2} d m_{2}^{2},
$$




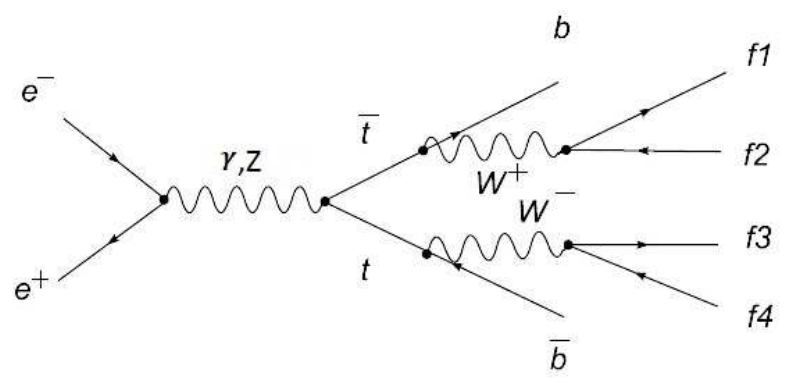

Figure 1: Feynman diagram of the process $e^{+} e^{-} \rightarrow t^{*} t^{*} \rightarrow b \bar{b} W^{+} W^{-} \rightarrow b \bar{b} 4 f$.

where $m_{0} \approx 2 m_{b}$ is the threshold value of the top mass, $\sigma\left(e^{+} e^{-} \rightarrow t\left(m_{1}\right) \bar{t}\left(m_{2}\right)\right)$ is the cross-section of top pair production with random masses $m_{1}$ and $m_{2}$. The $\rho_{t}(m)$ is the probability density in the Lorentzian form [9]

$$
\rho_{t}(m)=\frac{1}{\pi} \frac{m \Gamma_{t}(m)}{\left(m^{2}-M_{t}^{2}\right)^{2}+m^{2} \Gamma_{t}^{2}(m)},
$$

where $\Gamma_{t}(m)$ is the total decay width of the top quark with mass $m$. The top quark decay mode $t \rightarrow b W$ has a branching ratio $\operatorname{Br}(t \rightarrow b W) \approx 0.999$, so formula (2.1) almost exactly describes the cascade process $e^{+} e^{-} \rightarrow t^{*} \bar{t}^{*} \rightarrow b \bar{b} W^{+} W^{-}$in the stable $W$-boson approximation. In order to take into account the instability of $W$-bosons we have to express the top quark width $\Gamma_{t}(m) \approx \Gamma(t \rightarrow b W)$ in Eq.(2.2) as a function of smeared boson mass $\Gamma\left(t \rightarrow b W\left(m_{W}\right)\right)$ with averaging over $m_{W}$. Thus, the model cross-section of the full inclusive process $e^{+} e^{-} \rightarrow t^{*} \bar{t}^{*} \rightarrow b \bar{b} W^{+} W^{-} \rightarrow b \bar{b} \sum_{f} 4 f$ has the following convolution form:

$$
\begin{array}{r}
\sigma\left(e^{+} e^{-} \rightarrow b \bar{b} \sum_{f} 4 f\right)=\int_{m_{0}^{2}}^{s} \int_{m_{0}^{2}}^{\left(\sqrt{s}-m_{1}\right)^{2}} \sigma\left(e^{+} e^{-} \rightarrow t\left(m_{1}\right) \bar{t}\left(m_{2}\right)\right) \\
\int_{\left(m_{0}-m_{b}\right)^{2}}^{\left(m_{1}-m_{b}\right)^{2}} \rho_{t}\left(m_{1}, m_{W^{+}}\right) \rho_{W}\left(m_{W^{+}}\right) d m_{W^{+}}^{2} \int_{\left(m_{0}-m_{b}\right)^{2}}^{\left(m_{2}-m_{b}\right)^{2}} \rho_{t}\left(m_{2}, m_{W^{-}}\right) \rho_{W}\left(m_{W^{-}}\right) d m_{W^{-}}^{2} d m_{1}^{2} d m_{2}^{2},
\end{array}
$$

where $\rho_{W}(m)$ is defined by Eq. (2.2).

The results of the SMUP model calculations are presented in Fig. 2. Here, the dotted line represents the cross-section of the top-pair production in the stable particle approximation (SPA). The dashed line is the cross-section incorporating the top mass smearing only, and the solid line gives the full mass smearing result, both top-quarks and $W$-bosons.

From Fig. 2, one can see that the contribution of the top quarks' FWEs is significant (up to a few percents in the near-threshold region), while the contribution of $W$-bosons' FWEs is small. The comparison of our results with ones in the standard perturbative treatment shows that deviations are typically very small. For instance, it was obtained in Ref. [11], that $\sigma\left(e^{+} e^{-} \rightarrow t^{*} \bar{t}^{*} \rightarrow b \bar{b} W^{+} W^{-}\right)$ for $\sqrt{s}=500 \mathrm{GeV}$ is equal to $629 \mathrm{fb}$ for $m_{t}=150 \mathrm{GeV}$ and $553 \mathrm{fb}$ for $m_{t}=180 \mathrm{GeV}$. For the same input data, we have obtained $630 f b$ and $554 f b$, respectively, which are in a good agreement with the result mentioned above. 


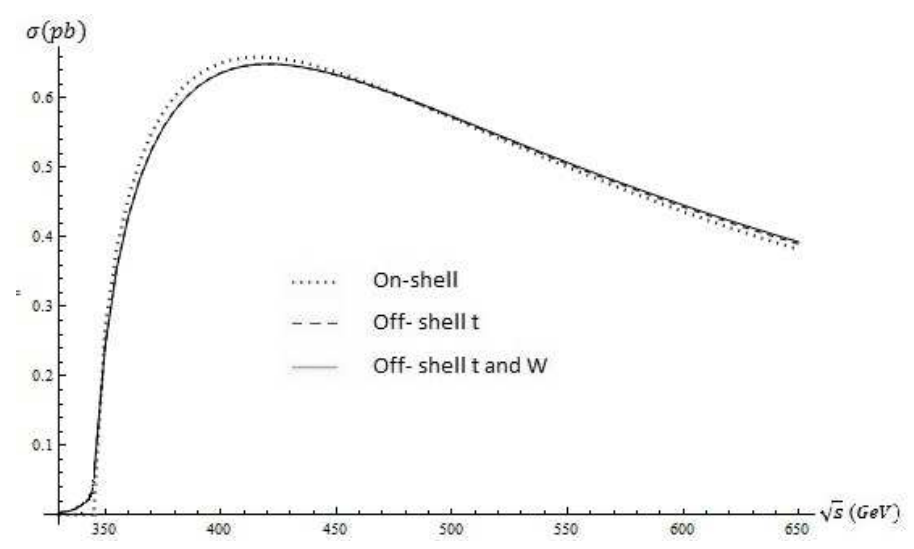

Figure 2: The cross-sections of the processes $e^{+} e^{-} \rightarrow t \bar{t}, e^{+} e^{-} \rightarrow b \bar{b} W^{+} W^{-}$and $e^{+} e^{-} \rightarrow b \bar{b} \sum_{f} 4 f$.

\section{Factorizable corrections to the cross-section}

In this Section, we describe the strategy of calculations and give the total cross-section including the NLO electro-weak and QCD corrections. In the frame of the SMUP model, the instability (or finite width) of unstable particles is accounted for by the smearing of their masses, i.e. by the probability density function $\rho(m)$. In turn, this function contains momentum dependent parameters $M(q)$ and $\Gamma(q)$. So, in that sense the corrections of self-energy type are already included at the "effective" tree level, and it is reasonable to use an effective couplings, such as running coupling, absorbing the major vertex-type corrections.

In our calculations we have used the following input data [12]:

$$
\begin{gathered}
\alpha\left(M_{Z}\right)=0.00781763, \quad \alpha_{s}\left(M_{Z}\right)=0.118, \quad \sin ^{2} \theta_{W}\left(M_{Z}\right)=\hat{s}_{Z}^{2}=0.2313 \\
M_{Z}=91.1876 \mathrm{GeV}, \quad M_{W}=80.399 \mathrm{GeV}, \quad M_{t}=172.9 \mathrm{GeV} .
\end{gathered}
$$

The running coupling constants $\alpha_{k}\left(Q^{2}\right), k=1,2,3$ were used in the one-loop approximation:

$$
\alpha_{k}\left(Q^{2}\right)=\frac{\alpha_{k}\left(M_{Z}\right)}{1-\left(\beta_{k} / 2 \pi\right) \ln \left(Q^{2} / M_{Z}^{2}\right)}, \beta_{k}=(4.1,-19 / 6,-7) .
$$

The cross-sections are calculated including the following corrections:

- Vertex and self-energy type corrections for stable particles are mainly included into running couplings (3.2).

- Self-energy corrections for unstable particles are included into the probability density function $\rho(m)$, which describes the smearing of UP's masses.

- Initial state radiation (ISR) is described by the photon radiation spectrum [13, 14], and the bremsstrahlung of $t$-quarks states - by vertex $Q$-dependent factor [15]. 


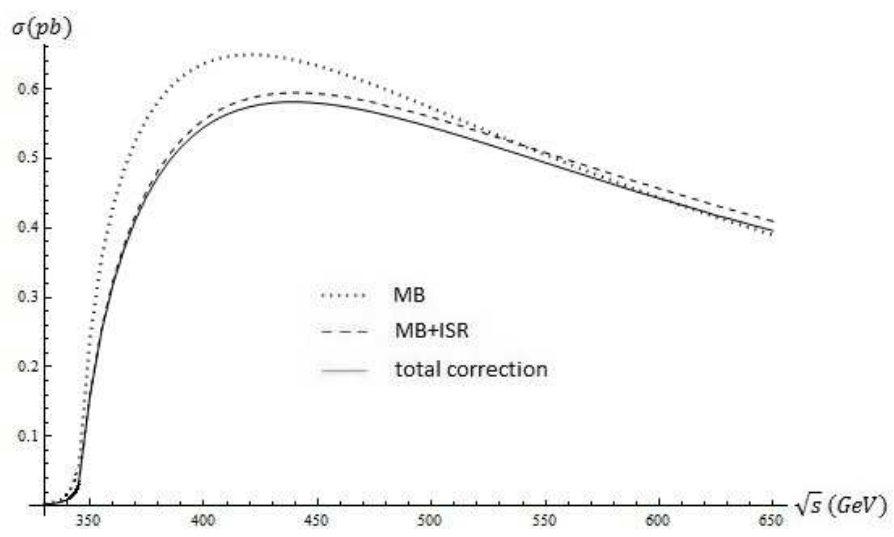

Figure 3: The higher order corrected cross-sections of the process $e^{+} e^{-} \rightarrow b \bar{b} \sum_{f} 4 f$.

- QCD corrections to the top production and decay are described by the vertex factor [15].

- Contribution of the box diagrams was roughly estimated at energy scales close to the threshold.

The corrected cross-sections of the inclusive process $e^{+} e^{-} \rightarrow t^{*} \bar{t}^{*} \rightarrow b \bar{b} \sum_{f} 4 f$ are shown in Fig. 3. There, the dotted line represents the Born cross-section, the dashed line - the cross-section with ISR and the solid line - the cross-section with total factorisable corrections (without box diagrams contribution). From the figure, one can see that the main contribution is given by ISR correction, which significantly reduces the cross-section in the near-threshold energy range and increases it at energy scales above $\sim 0.6 \mathrm{TeV}$. At large energies $(\sqrt{s}>0.5 \mathrm{TeV})$ the contribution of EW and QCD corrections becomes significant and has to be properly taken into account.

\section{The cross-section of the exclusive process}

In order to get the cross-section of the exclusive process $e^{+} e^{-} \rightarrow t^{*} \bar{t}^{*} \rightarrow b \bar{b} f_{1} f_{2} f_{3} f_{4}$ we can include the corresponding branching ratios $\operatorname{Br}\left(W \rightarrow f_{1} f_{2}\right)$ and $\operatorname{Br}\left(W \rightarrow f_{3} f_{4}\right)$. Acting this way we obtain

$$
\sigma\left(e^{+} e^{-} \rightarrow b \bar{b} f_{1} f_{2} f_{3} f_{4}\right)=\sigma\left(e^{+} e^{-} \rightarrow b \bar{b} \sum_{f} 4 f\right) \operatorname{Br}\left(W \rightarrow f_{1} f_{2}\right) \operatorname{Br}\left(W \rightarrow f_{3} f_{4}\right)
$$

The expressions for the branchings fractions $\operatorname{Br}\left(W \rightarrow f_{1} f_{2}\right)$ were considered in detail in Ref. [15]). Here, we use very simple but sufficiently precise formulae which incorporate QCD corrections:

$$
\operatorname{Br}\left(W \rightarrow l \bar{v}_{l}\right)=\frac{1}{9\left(1+2 \alpha_{s}\left(M_{Z}\right) / 3 \pi\right)}, \operatorname{Br}\left(W \rightarrow u_{i} \bar{d}_{k}\right)=\frac{\left|V_{i k}\right|^{2}\left(1+\alpha_{s}\left(M_{Z}\right) / \pi\right)}{3\left(1+2 \alpha_{s}\left(M_{Z}\right) / 3 \pi\right)},
$$

where $V_{i k}$ are elements of the Cabibbo-Kobayashi-Maskawa matrix. We, also, employ the QCD corrected expression for the top quark width $[15,16]$ :

$$
\Gamma(t \rightarrow b W)=\frac{1}{16} \alpha_{2}\left(m_{t}\right)\left|V_{t b}\right|^{2} \eta_{Q C D} m_{t} f\left(m_{t}, m_{W}, m_{b}\right),
$$


where

$$
\begin{gathered}
f\left(m_{t}, m_{W}, m_{b}\right)=\lambda\left(m_{b}^{2}, m_{W}^{2} ; m_{t}^{2}\right)\left(\frac{\left(m_{t}^{2}-m_{b}^{2}\right)^{2}}{m_{t}^{2} m_{W}^{2}}+\frac{m_{t}^{2}+m_{b}^{2}-2 m_{W}^{2}}{m_{t}^{2}}\right) ; \\
\lambda\left(m_{b}^{2}, m_{W}^{2} ; m_{t}^{2}\right)=\left(1-2 \frac{m_{b}^{2}+m_{W}^{2}}{m_{t}^{2}}+\frac{\left(m_{W}^{2}-m_{b}^{2}\right)^{2}}{m_{t}^{4}}\right)^{1 / 2} ; \\
\eta_{Q C D}=1-\frac{2 \alpha_{s}\left(M_{t}\right)}{3 \pi}\left(\frac{2 \pi^{2}}{3}-\frac{5}{2}\right) .
\end{gathered}
$$

Using Eqs. (4.1)-(4.3) we can calculate exclusive cross-section for an arbitrary six-fermion final state $\left(b \bar{b} f_{1} f_{2} f_{3} f_{4}\right)$. Such calculations were performed in standard approach for the case of $\left(b \bar{b} \mu^{+} v_{\mu} \mu^{-} \bar{v}_{\mu}\right)$ and $\left(b \bar{b} \mu^{+} v_{\mu} d \bar{u}\right)$ final states in Ref. [8]. In Table 4 the cross-sections are given for the two distinct reactions

$$
e^{+} e^{-} \rightarrow b \bar{b} \mu^{+} v_{\mu} \mu^{-} \bar{v}_{\mu}, e^{+} e^{-} \rightarrow b \bar{b} \mu^{+} v_{\mu} d \bar{u}
$$

for the energies $\sqrt{s}=430,500,1000, \mathrm{GeV}$. The results in the Born approximation are shown in the second column. The cross-sections with separate ISR and factorizable EW corrections are presented in the third and forth columns, respectively, and the cross-section with both FEWC and ISR corrections included - in the fifth column. All values are calculated with the kinematical cuts mentioned above (see also Ref. [7]).

\begin{tabular}{|c||c|c|c|c|}
\hline \multicolumn{1}{|c|}{$\sqrt{s}, \mathrm{GeV}$} & $\sigma_{\text {Born }}^{t^{*} \bar{t}^{*}}$ & $\sigma_{\text {Born }+ \text { ISR }}$ & $\sigma_{\text {Born+FEWC }}$ & $\sigma_{\text {Born+ISR+FEWC }}$ \\
\hline \multicolumn{5}{|c|}{$e^{+} e^{-} \rightarrow b v_{\mu} \mu^{+} \bar{b} \mu^{-} \bar{v}_{\mu}$, Ref. [8] } \\
\hline 430 & $5.8642(45)$ & $5.2919(91)$ & $5.6884(55)$ & $5.0978(53)$ \\
500 & $5.2849(43)$ & $5.0997(51)$ & $4.9909(49)$ & $4.8085(48)$ \\
1000 & $1.6369(15)$ & $1.8320(18)$ & $1.4243(14)$ & $1.6110(16)$ \\
\hline \multicolumn{5}{|c|}{$e^{+} e^{-} \rightarrow b v_{\mu} \mu^{+} \bar{b} \mu^{-} \bar{v}_{\mu}$, this work } \\
\hline 430 & 5.86476 & 5.27613 & 5.77727 & 5.19941 \\
500 & 5.27352 & 5.08651 & 5.18407 & 5.00291 \\
1000 & 1.63061 & 1.83508 & 1.58925 & 1.79079 \\
\hline \hline \multicolumn{5}{|c|}{$e^{+} e^{-} \rightarrow b v_{\mu} \mu^{+} \bar{b} d \bar{u}$, Ref. [8] } \\
\hline 430 & $17.592(13)$ & $15.857(20)$ & $17.052(16)$ & $15.283(16)$ \\
500 & $15.855(13)$ & $15.311(15)$ & $14.977(16)$ & $14.438(14)$ \\
1000 & $4.9106(46)$ & $5.4949(55)$ & $4.2697(40)$ & $4.8287(47)$ \\
\hline \multicolumn{5}{|c|}{$e^{+} e^{-} \rightarrow b v_{\mu} \mu^{+} \bar{b} d \bar{u}$, this work } \\
\hline 430 & 17.8163 & 16.0351 & 17.5540 & 15.8019 \\
500 & 16.0203 & 15.4517 & 15.7516 & 15.1979 \\
1000 & 4.95397 & 5.57465 & 4.82889 & 5.44011 \\
\hline
\end{tabular}

Table 1: Comparison of the exclusive cross-sections of Ref. [8] and obtained in the present work.

From Table 4, it follows that the differences of the model and standard Born cross-sections are of an order of 0.1 percent and an account of ISR increases it only slightly. The situation becomes worse, when we take into account the all corrections. The deviations increase and become up to a few percents. This discrepancy is caused by the fact that in Ref. [8] an additional contribution from the non-signal (background) diagrams was included while we consider the signal contribution only. Moreover, we do not include the contribution of the box diagrams which becomes very important at large energies far from the threshold. According to estimations in the framework of the standard perturbative treatment, the box diagrams contribution is of an order of a few percents 
in the near-threshold energy range. Rough estimations in the framework of the SMUP model give the box contribution equal to $1.5-2$ percents in the energy region under consideration, and these estimations decrease the deviations.

\section{Conclusion}

The production of a $t \bar{t}$ pair and its subsequent decay into six fermion final states in $e^{+} e^{-}$ annihilation has been previously analyzed within the standard treatment in a vast literature. In this work, we performed the corresponding analysis in the framework of SMUP model. So far, this approach was applied mainly to the gauge boson production, where the structure of the model boson propagators was tested successfully. In the present work, we have tested the structure of the model fermion propagator. It was shown that the results of Born-level calculations are in a good agreement with the standard ones, providing the applicability of the SMUP approach to the top-quark production processes.

The SMUP model provides simple analytical expressions for the cross-sections of inclusive and exclusive processes of top pair production and its subsequent decay. It is a convenient and simple instrument for description of complicated multi-step processes with unstable particles participation. The precision of the approach at the tree level is of an order of 0.1 percent. The method gives a possibility to include, in principle, all factorizable corrections. Our approach is useful in a preliminary analysis of complicated processes with intermediate top quark exchanges within the Standard Model and beyond.

\section{References}

[1] D. Chakraborty, J. Konigsberg and D. L. Rainwater, Ann. Rev. Nucl. Part. Sci. 53, (2003) 301.

[2] T. Han, Int. J. Mod. Phys. A, 23, (2008) 4107.

[3] W. Bernreuther, J. Phys. G 35, (2008) 083001.

[4] K. Kolodziej, Eur. Phys. J. C 23, (2002) 471.

[5] F. Yuasa et al., Phys. Lett. B 414, (1997) 178.

[6] E. Accomando et al., Nucl. Phys. B 512, (1998) 19.

[7] F. Gangemi et al., Nucl. Phys. B 559, (1999) 3.

[8] K. Kolodziej et al., Eur. Phys. J. C 46, (2006) 357.

[9] V. I. Kuksa, Int. J. Mod. Phys. A 24, (2009) 1185.

[10] V. I. Kuksa and N. I. Volchanskiy, Int. J. Mod. Phys. A 25, (2010) 2049.

[11] A. Ballestrero et al., Phys. Lett. B 333, (1994) 434.

[12] K. Nakamura et al. (Particle Data Group), J. Phys. G 37, (2010) 075021.

[13] J. Fleisher et al., Phys. Rev. D 47, (1993) 830.

[14] W. Beenakker et al., in Physics at LEP2, eds. G. Altarelli, T. Sjöstrand and F. Zwirner (CERN 96-01, Geneva, 1996), Vol. 1, p. 79.

[15] A. Denner, Fortschr. Phys. 41, (1993) 307.

[16] Guo Lei et al., Phys. Lett. B 662, (2008) 150. 